\title{
Weakly Segregated Smectic C Lamellar Clusters in Blends of Rods and Rod-Coil Block Copolymers
}

\author{
Nicolas Sary, ${ }^{\dagger}$ Raffaele Mezzenga, ${ }^{*}, \dagger, \downarrow$ Cyril Brochon,,$\S$ Georges Hadziioannou, ${ }^{\S}$ and \\ Janne Ruokolainen ${ }^{\perp}$ \\ Department of Physics and Fribourg Center for Nanomaterials, University of Fribourg, Ch. Musée 3, \\ CH-1700, Fribourg, Switzerland; Nestlé Research Center, Vers-Chez-Les-Blanc, 1000 Lausanne 26, \\ Switzerland; Laboratoire d'Ingénierie des Polymères pour les Hautes Technologies, UMR 7165, \\ Université Louis Pasteur, Ecole Européenne de Chimie, Polymères et Matériaux, 25, Rue Becquerel, \\ 67087 Strasbourg, France; and Physics Laboratory, Helsinki University of Technology, \\ Helsinki 02015, Finland
}

\begin{abstract}
The morphology arising from the self-assembly of $\pi$-conjugated poly(diethylhexyloxy-pphenylenevinylene) rod homopolymers (PPV) and poly(diethylhexyloxy-p-phenylenevinylene)-polystyrene (PPVPS) rod-coil block copolymers is described. Two PPV-PS block copolymers, one with low rod volume fraction $(\sim 17 \%)$ and the other with high volume fraction $(\sim 50 \%)$, were synthesized by convergent anionic polymerization and atom transfer radical polymerization, respectively. In the first case, given the low volume fraction of the rod block, the pure asymmetrical rod-coil block copolymer formed an isotropic homogeneous phase. However, ordered clusters of alternating PS and PPV domains with characteristic length of the order of several micrometers appeared when PPV rod homopolymers were blended to the PS-PPV diblock. Furthermore, the long-range order of the clusters as well as their volume fraction could be greatly increased when the symmetric rod-coil PPV-PS was blended to PPV homopolymer. Tomographic reconstruction from transmission electron micrographs allowed demonstrating that the clusters were organized in lamellar phase with well-defined width for both the intercalated PS and PPV domains, while wide-angle X-ray scattering showed that within the PPV domains the PPV blocks and PPV homopolymer rods were closely packed. The study of the spacing widths of the PPV and PS domains showed that clusters are organized in a smectic $\mathrm{C}$ configuration with large tilt angles of the rods $\left(54^{\circ}\right)$ and stretching of the coil blocks which is typical of weakly segregated block copolymers organized in a lamellar phase. The stability of the rod-to-rod interaction peak at high temperatures $\left(190^{\circ} \mathrm{C}\right)$, well beyond the order-disorder transition temperature of the clusters $\left(130^{\circ} \mathrm{C}\right)$, suggests that the (i) aggregation of the rods is mediated by $\pi-\pi$ interactions and (ii) the clusters are thermodynamically stable structures. The energetic driving force toward the formation of these clusters is discussed in the last part of this work.
\end{abstract}

\section{Introduction}

A large number of experimental and theoretical studies have been devoted during the past 25 years to the understanding of microphase separation of flexible diblock copolymers, also known as coil-coil block copolymers, yielding to an extensive knowledge of their phase diagram. ${ }^{1-3}$ In the case of a coilcoil block copolymer, the equilibrium microseparated phase, which can either be spherical, hexagonal, bicontinuous gyroid, or lamellar, will depend on the volume ratio of the two blocks as well as on the segregation parameter, expressed as the FloryHuggins interaction parameter times the polymerization degree $(\chi N)$. Conformational asymmetry between the two blocks has also been shown to have an effect. ${ }^{4}$ It is also well-known that the structure of coil-coil block copolymer may be changed by swelling either phase upon blending the diblock with limited amounts of any of the corresponding homopolymers. ${ }^{5}$ However, this route to control the morphology has severe restrictions, as the homopolymer must have a low molecular weight and the amount which can be added is limited to a very low volume

\footnotetext{
* To whom correspondence should be addressed: e-mail raffaele. mezzenga@unifr.ch, Tel +4 4126300 9066, Fax + 4126300 9747; e-mail raffaele.mezzenga@rdls.nestle.com, Tel + 4121785 8078, Fax + 4121 7858554 .

University of Fribourg.

Nestlé Research Center.

$\S$ Université Louis Pasteur.

${ }^{\perp}$ Helsinki University of Technology.
}

fraction of the block copolymer, ${ }^{6}$ beyond which macrophase separation occurs.

Rod-coil block copolymers are a different class of block copolymers in which the polymer forming one of the two blocks has a persistence length of the order of the end-to-end distance or greater. The long persistence length can be provided either by the formation of $\alpha$-helices, such as in the case of polypeptides, or by $\pi$-electronic cloud delocalization over many carbon atoms in the case of $\pi$-conjugated polymers. Similarly to the polypeptides, studies on rod-coil block copolymers with $\pi$-conjugated blocks have been expanding by remarkable advances on the synthetic routes followed to design this class of block copolymers. In the divergent way, copolymers are obtained starting from a conjugated macroinitiator by living polymerization of the coil block. The use of a conjugated macroinitiator has been already successfully used for the synthesis of rod-coil copolymers with poly( $p$-phenylenevinylene) ${ }^{7-9}$ polythiophene, ${ }^{10,11}$ polyfluorene, ${ }^{12,13}$ poly(thienylenevinylene), ${ }^{14}$ or oligomers. ${ }^{15,16}$ An alternative route consists of successively polymerizing by living anionic polymerization two coil blocks where one is a precursor of the rod segment. ${ }^{17}$ This way is however very restrictive for the choice of the rod block, and some permanent defects in the conjugation can occur. Most of the conjugated rod-coil copolymers have thus been obtained by convergent way, either by a simple condensation reaction between an end-functionalized coil 
polymer and the rod block ${ }^{18-22}$ or by quenching a living coil polymer, synthesized by anionic polymerization, on an endfunctionalized conjugated oligomer. ${ }^{23-25}$ This last approach leads to very-well controlled block copolymer since anionic living polymerization is an established technique to yield welldefined homopolymers and block copolymers. ${ }^{26}$

The phase diagram of rod-coil block copolymers is by far less universal and less well-understood than that of the coilcoil block copolymers, and no systematic studies have been reported for rod-coil plus rod blends. The presence of a rigid block introduces liquid crystalline behavior, which can compete with the classical microseparation mechanisms encountered in coil-coil block copolymers. Within a segregated phase of the rod component, rods may be aligned along a common director, forming for example layered smectic phases. ${ }^{27}$ Equivalently, an isotropic rod-coil diblock may retain orientation of the rod and thus provide birefringency to the material. ${ }^{28}$ It has been reported that at low rod weight fraction phases typical of coil-coil systems can be observed for rod-coil block copolymers. ${ }^{29-31}$ At large rod weight fraction, when liquid crystallinity dominates, nematic and smectic phases are the most frequently encountered phases. ${ }^{27,32,33}$ However other phases, which strongly differ from coil-coil block copolymer systems, have been theoretically postulated $^{34}$ or observed experimentally. ${ }^{35,36}$ In general, the rigidity of the rod block and rod-rod interactions, both being intimately related to the nature of the rod considered, are expected to have great influence on the molecular packing and thus on the structure of the thermodynamically stable phases.

The rod block involved in the present work, poly(diethylhexyloxy-p-phenylenevinylene) (PPV), is studied not only from a fundamental point of view but also as a precursor for rodcoil block copolymers to be used in the field of polymeric photovoltaic devices, such as $\mathrm{PPV}-\mathrm{P}\left(\mathrm{S}-\mathrm{CMSC}_{60}\right)$ used in solar cells. ${ }^{7}$ Semiconducting polymers for photovoltaic applications require that the material on the one hand is able to generate charges upon light exposure and on the other can support bipolar charge transport, allowing the extraction of the generated charges via the electrodes. Considering the low dielectric constant difference in organic materials, excitons rather than free charge carriers are created by photoexcitation. ${ }^{37}$ These excitons explore a space of the order of a nanometer, associated with their lifetime, before decaying to the ground state. The separation of these excitons in charges is promoted at the interface between electron acceptor and electron donor phases. Moreover, the average size of the microphases needs to be of the order of a nanometer to achieve quantitative and efficient exciton dissociation. Therefore, in order to achieve the highest efficiency in the charge separation process, it becomes crucial to finely control the interface, and hence the whole microstructure of the photovoltaic active media. This is in principle possible to be realized by electron donor-electron acceptor block copolymers self-assemblying into structures such as cylindrical, lamellar, or gyroidal.

In view of photovoltaic systems with a block copolymer as active material, where one block act as acceptor of charges and the other as donor, this paper provides the understanding and the control for the creation of interfaces and ordered lamellar microphases by blending donor $\pi$-conjugated PPV rods and PPV-PS rod-coil block copolymers which, alone, are either sufficiently asymmetric to form a fully isotropic phase or a nonpercolating disordered cluster-like structure. In what follows, we present an original morphology based on clustered lamellar structures occurring upon blending PS-PPV and PPV homopolymer. We discuss in detail the molecular organization of
Scheme 1. Main Steps Followed for the Synthesis of PPV-b-PS Block Copolymer
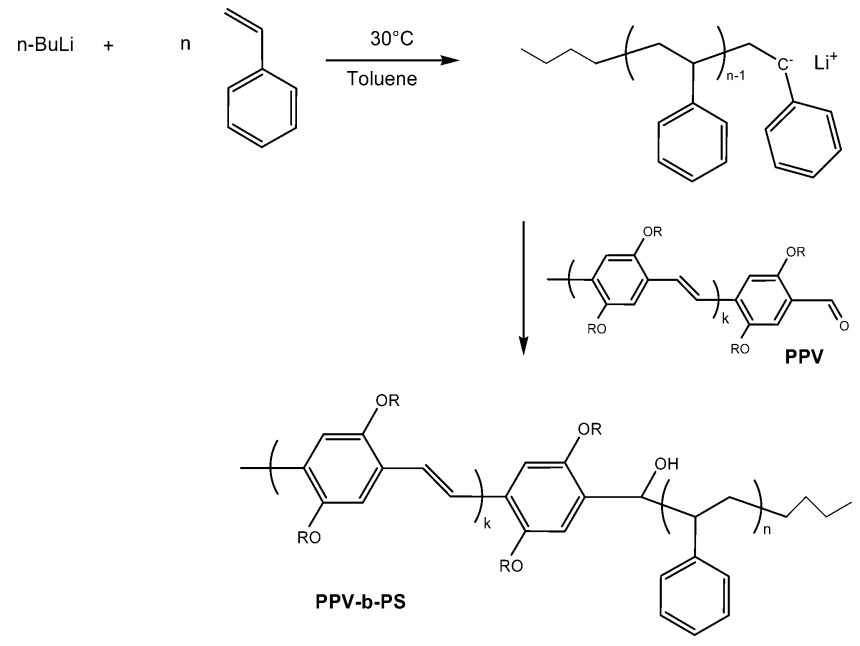

the rod and coil domains forming these structures, and we argue on the energetic driving force allowing the formation of these structures.

\section{Experimental Section}

Materials. Butyllithium (1.6 M in hexane), used as initiator for anionic polymerization, was purchased from Aldrich.

Styrene (Aldrich) was distilled on calcium hydride just before use. Toluene was distilled on sodium/benzophenoe just before use.

PPV was synthesized according to a procedure published elsewhere. ${ }^{38}$ Afterward, PPV was purified by column chromatography on a silica gel (cyclohexane with 5\% of dichloromethane), and its polydispersity index (PID) was measured at 1.2 on gel permeation chromatography using polystyrene standards. The number-average molecular weight, $M_{\mathrm{n}}$, and number of units $(n)$ were determined by nuclear magnetic resonance (NMR) $\left(M_{\mathrm{n}}=3400\right.$ $\mathrm{g} / \mathrm{mol}, n=9)$. PPV was dried by iterative azeotropic distillation in dried toluene just before use.

GPC and NMR Characterizations. ${ }^{1}$ NMR spectra were recorded on a Bruker 300 UltrashieldTM 300 MHz NMR spectrometer, with an internal lock on the $2 \mathrm{H}$ signal of the solvent $\left(\mathrm{CDCl}_{3}\right.$ or acetone- $d_{6}$ ).

GPC measurements were performed in THF (HPLC grade) with two PL-gel $5 \mu$ mixed-C, a $5 \mu 100 \AA$, and a $5 \mu$ Guard column in a Shimadzu LC-10AD liquid chromatograph equipped with a Shimadzu RID-10A refractive index detector and a Shimadzu SPPM10A diode array (UV) detector.

Rod-Coil Block Copolymer Synthesis. Anionic. Anionic polymerization with convergent route was followed in order to synthesize PPV- $b$-PS block (PS17K-PPV) copolymer. Welldefined aldehyde end-functionalized PPV ${ }^{38}$ was used as a quencher for the anionic polymerization of styrene (Scheme 1). This method is well-known ${ }^{23,25}$ and leads to well-defined block copolymers. In order to prevent a remaining fraction of PPV homopolymer in the final product, an amount of PPV corresponding to less than the stochiometric ratio $(0.95)$ was used as a quencher.

In a Schlenck flask sealed with a rubber septum, $1.4 \mathrm{~mL}$ of styrene $(1.35 \mathrm{mmol})$ and $10 \mathrm{~mL}$ of toluene were added under argon. The solution was degassed by three freeze-pump-thaw cycles. Butyllithium solution was added dropwise until the appearance of the red color. Then, $100 \mu \mathrm{L}$ of butyllithium solution $(0.16 \mathrm{mmol})$ was added. After $30 \mathrm{~min}$ at $30^{\circ} \mathrm{C}$, a solution of $307 \mathrm{mg}$ of PPV $(0.09 \mathrm{mmol})$ in $2 \mathrm{~mL}$ of dry toluene was added. After $10 \mathrm{~min}$, the reaction was quenched with $2 \mathrm{~mL}$ of methanol and then $1 \mathrm{~mL}$ of acidified methanol. Organic phase was washed three times with water, and then the copolymer was separated from residual homopolymer by chromatography on a silica gel (cyclohexane/ dichloromethane: 40/60). Finally, the block copolymer was precipitated in methanol (with $10 \%$ of water) and characterized by 
GPC with PS standards. An average total number molecular weight, $M_{\mathrm{n}}$, of $22200 \mathrm{~g} / \mathrm{mol}$ was measured, corresponding to a PS block of $16600 \mathrm{~g} / \mathrm{mol}$ and PPV block composed of 9 units. The PDI of the diblock copolymer (identified as PS17K-PPV in the following) was measured at 1.34 .

Atom Transfer Radical Polymerization. PPV was modified with bromomethylpropionyle bromide to form a macroinitiator. $61 \mathrm{mg}$ ( $0.43 \mathrm{mmol})$ of copper bromide (I) were complexed by $145 \mu \mathrm{L}$ $(0.53 \mathrm{mmol})$ of $1,1,4,7,10,10$-hexamethyltriethylenetetramine in 5.5 $\mathrm{g}$ of toluene. $5.5 \mathrm{~g}$ of styrene and $1.0 \mathrm{~g}$ of PPV-Br were then added to the solution. The polymerization was performed in a freezedried sealed tube. The diblock copolymer obtained (referred as PS3K-PPV) had an average total number molecular weight of 6400 $\mathrm{g} / \mathrm{mol}$ and PDI $=1.45$ and a PPV block made up of an average of 6.5 units. More details will be provided in an upcoming article.

Morphological Investigation. Samples Preparation. For consistency, all experiments on each specific system were performed on the same synthesis batch. PPV homopolymer used for the blends was obtained from the same batch used for the block copolymer synthesis. Densities of PPV and PS were 0.988 and $1.05 \mathrm{~g} / \mathrm{cm}^{3}$, respectively.

Three distinct solutions of 2 wt $\%$ of PS17K-PPV, PS3K-PPV, and PPV were prepared in choloroform (Acros 99 puriss) and then mixed in various proportions. The mixture was sonicated for $1 \mathrm{~h}$. TEM samples were prepared by evaporating the solvent at $20^{\circ} \mathrm{C}$ under vacuum $\left(10^{-3}\right.$ bar $)$.

Annealing Procedure. Owing to the moderate contrast to smallangle X-ray scattering and the low concentration of clusters (in the case of PS17K-PPV), the order-disorder transition temperature of the clusters observed could not be directly established by X-ray diffraction. Thus, a well-defined thermal cycle was followed in order to erase the thermal history of the samples and to maintain the blend in the temperature region comprised between the glass transition temperature of PS, $100{ }^{\circ} \mathrm{C}$, and the potential orderdisorder transition temperature of mesophases. All samples were annealed in a high vacuum $\left(5 \times 10^{-12}\right.$ bar $)$ oven according to the following procedure: $2 \mathrm{~h}$ at $220{ }^{\circ} \mathrm{C}, 12 \mathrm{~h}$ at $180{ }^{\circ} \mathrm{C}, 12 \mathrm{~h}$ at 160 ${ }^{\circ} \mathrm{C}, 12 \mathrm{~h}$ at $140{ }^{\circ} \mathrm{C}$, and finally $12 \mathrm{~h}$ at $120{ }^{\circ} \mathrm{C}$.

The order-disorder transition temperature of the clusters $\left(T_{\mathrm{ODT}}\right)$ was determined following the following procedure: samples were annealed every $20^{\circ} \mathrm{C}$ at temperatures ranging from 180 to $120^{\circ} \mathrm{C}$ for $48 \mathrm{~h}$ at $5 \times 10^{-12}$ bar; then, while maintaining the annealing temperature, the oven was filled by nitrogen up to atmosphere pressure and opened. The samples were then rapidly quenched in liquid nitrogen, collected, and investigated by TEM to establish whether the clusters were molten or not at all the specific temperatures investigated. Since temperatures below the glass transition of the system are rapidly achieved, this procedure quenches the morphology in the thermodynamic state reached at the annealing temperature and proved to be the most efficient way to detect $T_{\mathrm{ODT}}$ in all the blends investigated.

Small- and Wide-Angle X-ray Scattering (SAXS and WAXS). Small- and wide-angle X-ray scattering (SAXS, WAXS) diffractograms were acquired on a Anton-Paar SAXSess instrument. About $10 \mathrm{mg}$ of blend was solvent-cast on a mica sheet. For measurements relative to high temperatures, in order to avoid oxidation of the polymer and attain thermodynamic equilibrium, the sample was previously annealed at fixed temperature $(190,200,230$, and 250 ${ }^{\circ} \mathrm{C}$ ) in the vacuum-annealing column. Then, samples were rapidly quenched in liquid nitrogen as described above. Finally, the sample was transferred to SAXS-WAXS facilities for the diffraction measurements.

Cross-Polarized Optical Microscopy. The polymer was solvent cast on a glass substrate from dichloromethane solution. The sample was observed with cross polarizers on a Leica DM IRD optical microscopes, using a $40 \times$ magnification.

Ultramicrotomy. All samples were embedded in a standard fourcomponent epoxy resin (46 wt \% Epon 812, 28 wt \% (dodecenylsuccinic anhydride) DDSA, 25\% (nadic methyl anhydride) NMA, $1 \%$ 2,4,6-tris(dimethylaminomethyl)phenol) catalyst. In order to avoid diffusion of the resin components into the sample, the resin

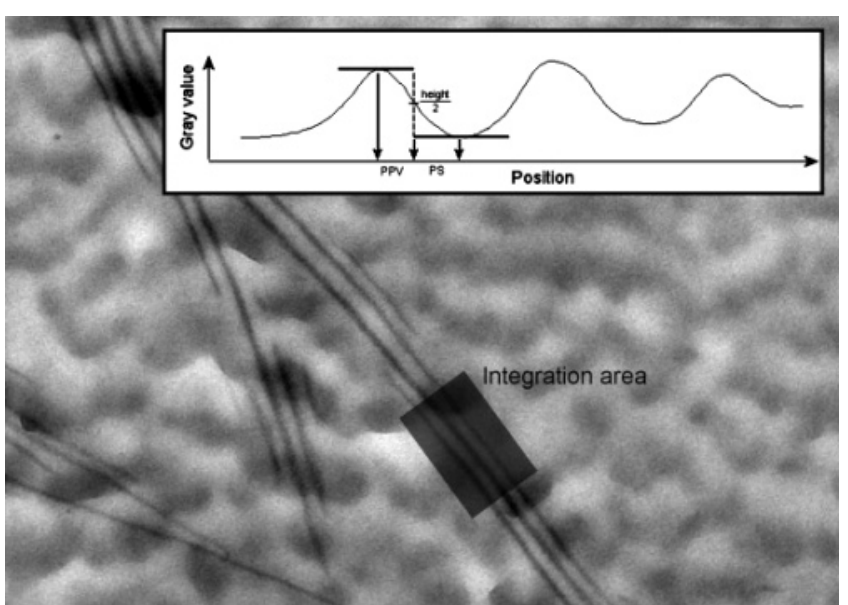

Figure 1. Determination of period and widths of both PPV and PS domains is performed using a home-developed software, which yields a histogram curve of the contrast over a desired area (here shown in dark gray). Maximum and minimum of this curve are automatically determined from the histogram and correspond to the center of PPV and PS domains, respectively. The domain boundaries are defined at the half-value of the contrast difference.

was precured $1 \mathrm{~h} 30 \mathrm{~min}$ at $80{ }^{\circ} \mathrm{C}$ before embedding the sample. The sample was finally embedded in the precured epoxy resin, which was then let curing for $8 \mathrm{~h}$ at $60{ }^{\circ} \mathrm{C}$.

The samples were ultramicrotomed on a Reichert-Jung microtome at room temperature. $60 \mathrm{~nm}$ thick sections were collected on 300 hexagonal mesh copper grids (EMS G300H-Cu).

Staining of the PPV phase was achieved by exposing collected sections to vapors of $\mathrm{OsO}_{4}(0.5 \mathrm{wt} \%$ salt in water) for $3 \mathrm{~h}$.

Transmission Electron Microscopy (TEM). Bright field imaging was performed on a CM100 Philips TEM operated at $80 \mathrm{kV}$ (emission 2). All images were acquired on a SIS Morada CCD camera.

Image Processing and Data Treatments. Domain widths and overall periods in heterogeneous phases were measured from TEM images by using a specially home-developed software enabling high-precision measurements. In order to correctly measure periods and width of individual domains, contrast histograms were recorded perpendicularly to domain interfaces, as shown in Figure 1. Domain boundaries were defined to be the points at which the contrast values correspond to the half of the difference between the maximum and minimum contrast values in the histogram (Figure 1). Average values were extracted only from high-contrast and sharp regions of clusters, which correspond to clusters orthogonally oriented to the microtomed section. Tilt experiments of the sections further confirmed the orthogonal alignment of the measured clusters to the observation plane. Tilt series alignments while operating TEM were performed and controlled by the EM3D software developed at Stanford University. ${ }^{39}$

\section{Results and Discussion}

The first system studied was the highly asymmetric PS17KPPV, where the rod fraction was present in a relatively low weight fraction, i.e., 0.17, which corresponds to a volume fraction of 0.18 . At this volume fraction in an equivalent coilcoil system, provided that $\chi N$ is high enough, one can expect isotropic, body-centered spheres or hexagonal phase, depending on the exact value of $\chi N$. On the rod-coil system considered, however, the expected phase will depend not only on the volume fraction and $\chi N$ but also on the Maier-Saupe constant, $\omega$, which expresses the rod-rod steric repulsion or more simply on the ratio $\omega / \chi$. At very large values of $\omega / \chi$, the liquid crystallinity provided by the rod blocks may drive the system into a smectic phase. ${ }^{32}$ Theoretically, for values of $\chi N$ below 30 and for $\omega / \chi$ below 8, the system is expected to be in either an isotropic 


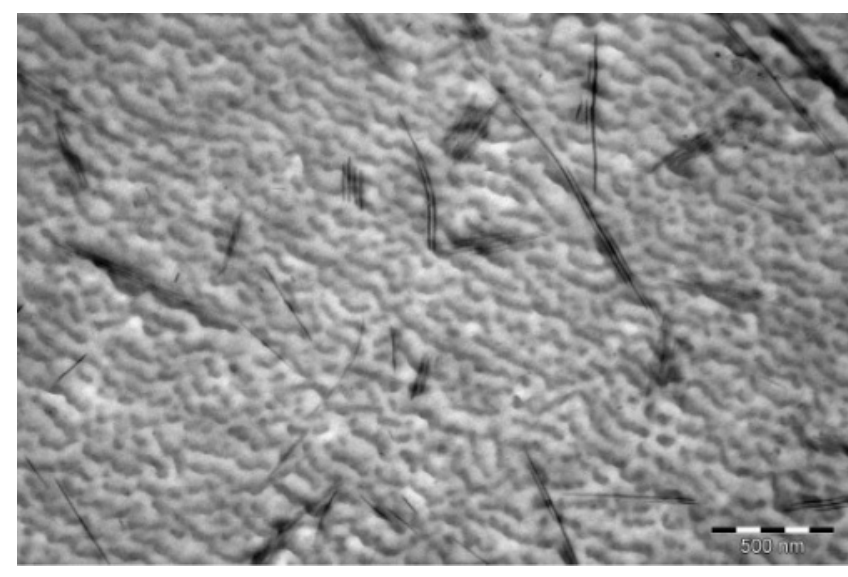

Figure 2. $\mathrm{OsO}_{4}$-stained TEM micrograph of a blend of $95 \mathrm{wt} \%$ PS$b$-PPV block copolymer and $5 \mathrm{wt} \%$ of PPV homopolymer rods, showing the onset of lamellar clusters formation.

phase $^{25,32}$ or a nematic phase. ${ }^{33}$ These findings have been already experimentally supported in PPV-polyisoprene systems, ${ }^{25}$ where, at large rod volume fraction, an increase of temperature (and thus decrease in $\chi N$ ) drives progressively the system from lamellar phase to nematic and isotropic phases, while at low rod volume fraction the system is always in the isotropic phase. The presence of an isotropic phase at large coil volume fraction is also confirmed in the present system, where a TEM micrograph for the synthesized PS17K-PPV rod-coil block copolymer shows no microsegregated morphologies (not shown here). Cross-polarized optical microscopy has allowed rulingout the presence of a birefringent nematic phase, thus confirming that the synthesized PS17K-PPV forms a fully isotropic homogeneous phase.

Figure 2 shows a TEM micrograph of the PS17K-PPV diblock, blended with 5\% weight fraction of PPV rod homopolymer. Elongated dark rodlike objects with characteristic length of typically one to several micrometers appear in the blend. They are mostly composed of PPV, since $\mathrm{OsO}_{4}$ selectively stains PPV. However, no distinction can be made at this point between the homopolymer and the block PPV. Furthermore, each PPV-rich domain appears to be aligned with several other (typically 4-5) and organized in the form of clusters characterized by a specific and well-defined pattern period. The appearance of these clusters is clearly related to the addition of PPV homopolymer, although the blending of PPV homopolymer to a PS homopolymer leads to very different morphologies. Indeed, as shown in Figure 3, the droplets of PPV macrophase separating from the PS matrix have not the typical spherical shape found in heterogeneous blends formed by nucleation and growth (NG), but rather an elliptic-like shape with a large aspect ratio and a termination angle. This suggests that the (i) the minimization of the total free energy of the systems accounts not only for the interfacial free energy associated with PPV/PS interfaces, but also additional contributions play a role, and (ii) that droplets originate by NG by subsequent stacking of PPV rods, which infer the observed angle. The overlap of $\pi$-conjugated planar bonds, which is known as being an important interaction in systems containing $\pi$-conjugated polymers, ${ }^{40}$ can provide an attractive potential controlling the NG mechanism observed in Figure 3. Similarly, when blending PPV homopolymer to PPV-PS block copolymer, the $\pi$-mediated rod-rod attraction can lead to an aggregation of rod homopolymers and rod blocks, increasing the effective volume fraction of the rod in the block copolymer and thus driving the system into the microsegregated region of the phase diagram. Therefore, ac-

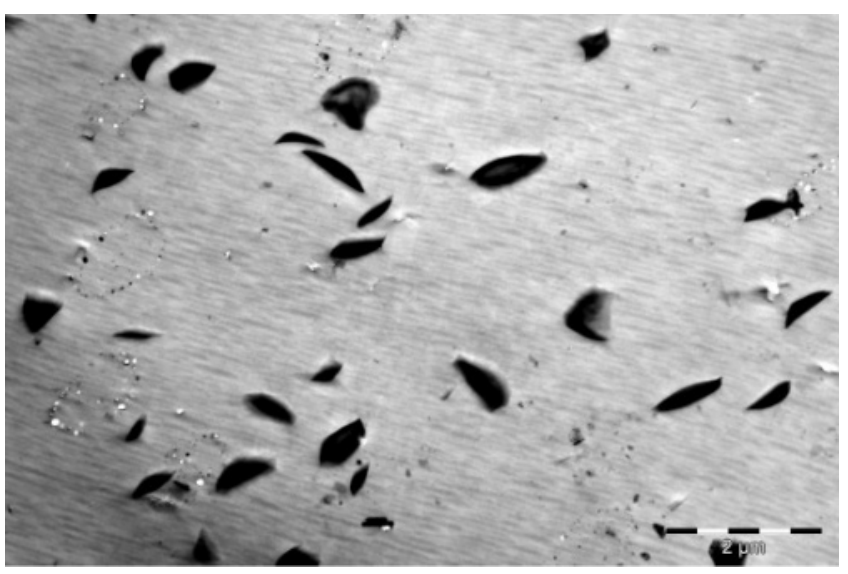

Figure 3. $\mathrm{OsO}_{4}$-stained TEM micrograph showing the structure of a blend formed by $90 \mathrm{wt} \%$ polystyrene homopolymer and $10 \mathrm{wt} \% \mathrm{PPV}$. The elongated shape of the dark PPV domains suggests that nucleation and growth occurs via subsequent stacking of PPV rods, rather than following an isotropic radial growth.

Size of PS and PPV domains

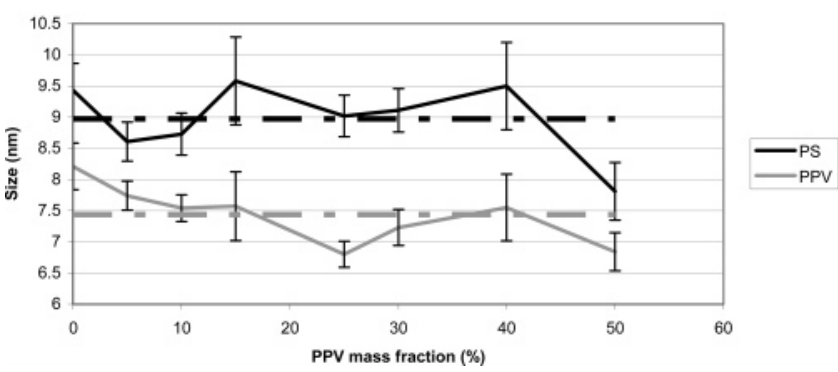

Figure 4. Variation of widths of PS and PPV domains measured by TEM as a function of blend's composition. Neither the cluster period nor the widths of PPV and PS domains vary significantly with respect to the weight fraction of the homopolymer PPV in the blend, although the number of clusters is increasing with PPV homopolymer volume fraction.

cording to this scenario, while the clear phase is essentially formed by PS, the PPV dark domain would be made of both homopolymer PPV and rod PPV, intercalated and strongly interacting.

In order to confirm this hypothesis and to better understand the exact structure of these PPV clusters in a PPV/PPV $17 \mathrm{~K}-$ PS blend, a number of parameters were varied and additional experiments performed.

Increasing PPV homopolymer fraction in the blend did not affect the shape, the characteristic length of the domains, the cluster period, and the domain size, within measuring accuracy as shown in Figure 4. Cluster density was, however, increased by raising the volume fraction of PPV. As homopolymer fraction was approaching $50 \mathrm{wt} \%$, clusters became more disordered and started to coexist with macrophase-separated PPV domains (Figure 5).

On the basis of the clusters pattern such as that shown in Figure 6 and available literature ${ }^{34}$ in the field of rod-coil block copolymers, one can imagine essentially three different types of structures for these clusters: rods, hockey pucks, and lamellae.

Because TEM images are two-dimensional projections of the real three-dimensional structure, the exact structure of the clusters cannot be worked out by using single TEM micrographs. Attempts have been made to use small-angle X-rays scattering to determine the form factor of the clusters, but owing to the low volume fraction of the clusters, combined with the very similar chemical composition of PS and PPV, and thus similar 


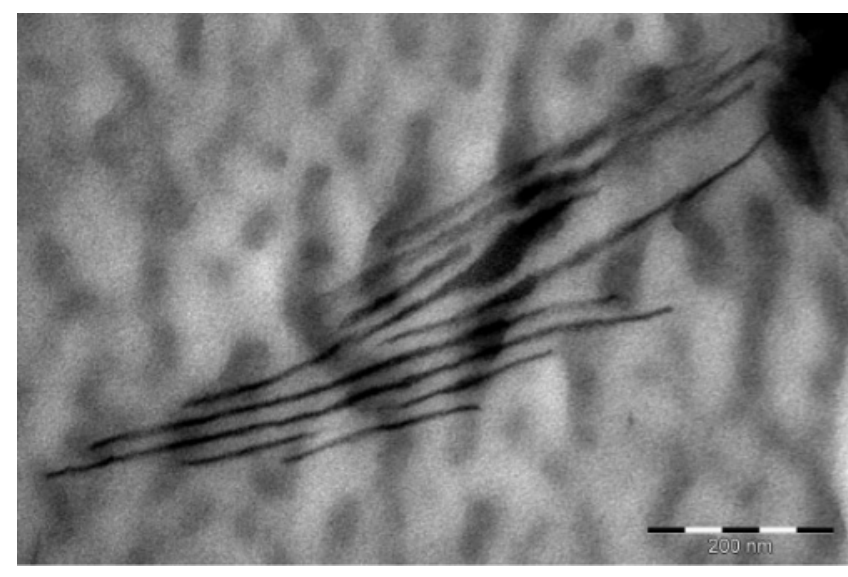

Figure 5. TEM micrographs showing the loss of order in the clusters when the fraction of homopolymer PPV is increased to $40 \%$.

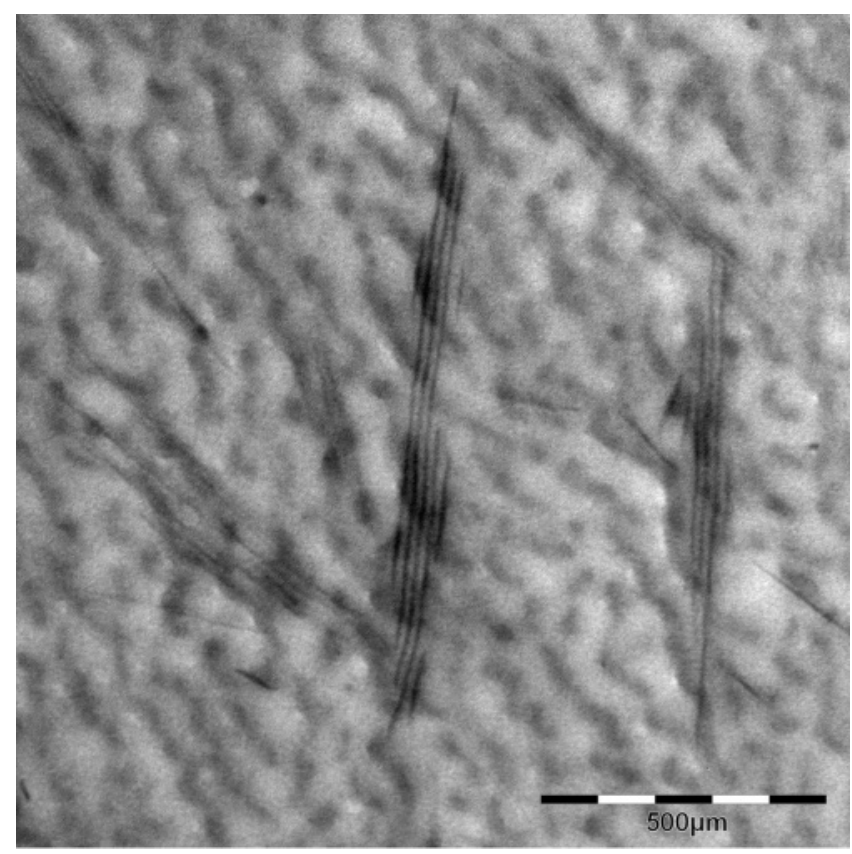

Figure 6. $\mathrm{OsO}_{4}$-stained TEM micrograph showing lamellar clusters in a blend of 90 wt \% PS- $b$-PPV block copolymer and $10 \mathrm{wt} \%$ of PPV homopolymer rods.

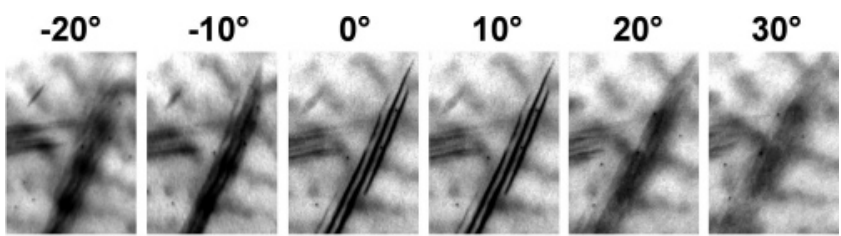

Figure 7. Electron microscopy tomographic sequence of a PPV/PS$b$-PPV cluster as a function of tilt angle (tilt axis is parallel to the vertical axis of the micrographs). The progressive loss of contrast as a function of tilt angle demonstrates the lamellar structure of the clusters over possible cylindrical or hockey puck shapes.

scattering lengths, contrast in SAXS was too low to provide any valuable information on cluster ordering. Therefore, TEM tomography was performed to determine the structure. By tilting the microtomed sections of the sample, one has access to the projection corresponding to virtually any desired angle, as shown in Figure 7 where the TEM image of one cluster is observed at six different tilting angles in a blend of $5 \mathrm{wt} \% \mathrm{PPV}$ in the PS17K-PPV matrix. On the basis of the evidence that strong contrast is lost as soon as the structure is not perpendicular to the projection plane, this allows one to conclude that the clusters
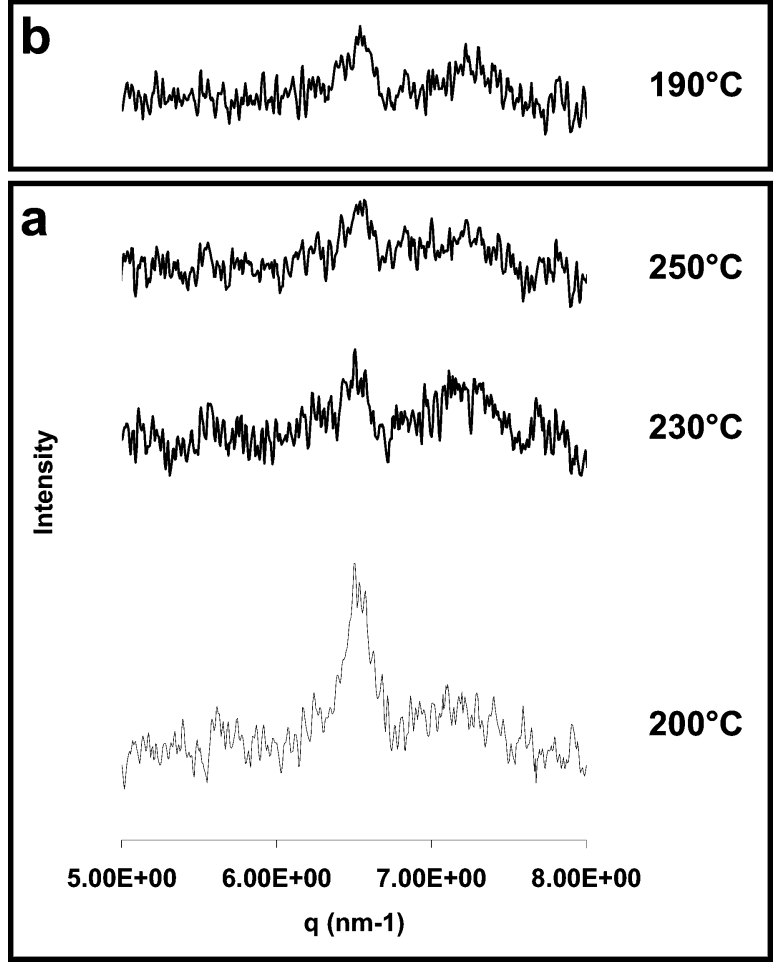

Figure 8. Wide-angle $X$-ray scattering diffractograms showing a single peak at $6.3 \mathrm{~nm}^{-1}$ corresponding to the rod-to-rod distance. (a) The interrod peak is stable up to to $250{ }^{\circ} \mathrm{C}$, at which temperature it starts to decrease due to thermal degradation. (b) The intensity is not recovered over cooling due to PPV chemical degradation at high temperatures.

are formed by lamellae. Furthermore, because one can tilt the section as much as needed to attain the maximum contrast, and thus orientation of the lamellae orthogonal to the projection plane, the spacing of both the clear PS and dark PPV domains can be accurately measured. This is another advantage over diffraction techniques, which, provided the contrast is sufficient, would yield only the overall lattice space of the clusters.

Wide-angle X-ray scattering, shown in Figure 8a, was performed to provide more insight into the way rods are packed within the dark PPV domains. While the PS17K-PPV block copolymer alone showed no peaks in WAXS diffractograms, a peak located at $6.53 \mathrm{~nm}^{-1}$ appeared on PPV/PS17K-PPV blends. Moreover, the rod-rod distance characteristic peak was sharp at $190{ }^{\circ} \mathrm{C}$ and is still present at $250{ }^{\circ} \mathrm{C}$. This peak, which in real space corresponds to a distance of $1 \mathrm{~nm}$, was attributed to the interspacing between rods, which need to optimize spacefilling requirements, side-chain steric hindrance, and $\pi$-stacking interactions. The most efficient way for the rods to pack fulfilling these requirements is to align along a common director, thus providing liquid crystallinity behavior to the PPV domains. A temperature increase up to $200{ }^{\circ} \mathrm{C}$ could not disrupt the rodrod interaction but certainly initiated the degradation of some of the PPV double bonds. This degradation is evident in Figure 8b: the WAXS characteristic peak of rod-rod interactions started disappearing at $230{ }^{\circ} \mathrm{C}$ and was not recovered when cooling the sample to $190{ }^{\circ} \mathrm{C}$.

The order-disorder transition temperature of the clusters, $T_{\mathrm{ODT}}$, was obtained by TEM quenching experiments and was found to be completely independent of the rod-rod interactions. Figure 9a shows a TEM picture taken on a sample annealed at $180{ }^{\circ} \mathrm{C}$ and rapidly quenched. As can be noted, PPV aggregated domains are visible, as confirmed by WAXS scattering data, although the ordered lamellar clusters have been completely melted. As temperature is progressively lowered, the number 

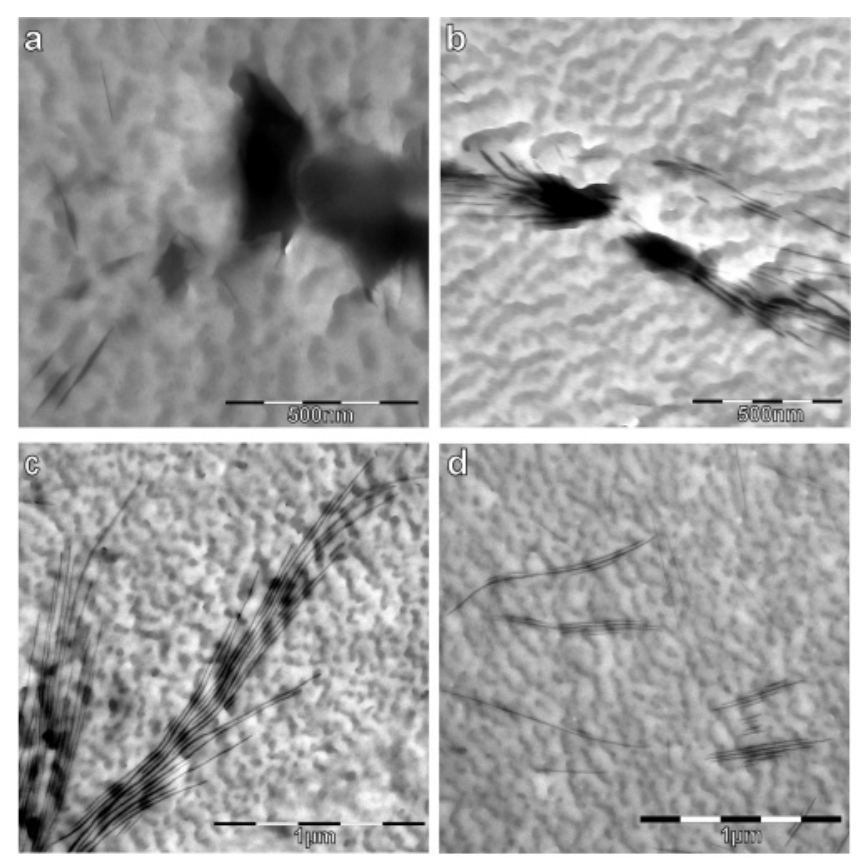

Figure 9. Determination of the lamellar clusters $T_{\mathrm{ODT}}$ by TEM analysis on samples rapidly quenched from the annealing temperature down to room temperature. Samples were annealed at the following temperatures: (a) $48 \mathrm{~h}$ at $180{ }^{\circ} \mathrm{C}$; (b) $48 \mathrm{~h}$ at $160^{\circ} \mathrm{C}$; (c) $48 \mathrm{~h}$ at $140{ }^{\circ} \mathrm{C}$; (d) $48 \mathrm{~h}$ at $120^{\circ} \mathrm{C}$. Based on the lamellar ordering appearing between 140 and $120^{\circ} \mathrm{C}$, the $T_{\mathrm{ODT}}$ is located at $\approx 130{ }^{\circ} \mathrm{C}$.

of aggregates start first to increase (Figure $9 \mathrm{~b}, 160{ }^{\circ} \mathrm{C}$ ), and then to organize in a rough lamellar organization (Figure 9c, $\left.140{ }^{\circ} \mathrm{C}\right)$, until when ordered lamellar domains are fully recovered at about $120{ }^{\circ} \mathrm{C}$ (Figure 9d). Therefore, according to these experiments, the $T_{\mathrm{ODT}}$ of the clusters can be located between 140 and $120{ }^{\circ} \mathrm{C}$, that is $\approx 130{ }^{\circ} \mathrm{C}$, which is lower than the melting temperature of the rod-rod interactions by more than $100{ }^{\circ} \mathrm{C}$. These results are important since demonstrate that the lamellar clusters are a thermodynamically stable phase which is not induced by the crystallization of the PPV rods.

Although WAXS data strongly support the alignment and close packing of homopolymer and rod PPV, no direct information is provided on the way layers of PPV and PS are organized within the clusters. Consistently with the TEM images and organization of rods, four different smectic phases can be envisaged to interpret the cluster structure: smectic $\mathrm{A}$ bilayer $\left(\mathrm{S}_{\mathrm{M}} \mathrm{A}_{2}\right)$, smectic $\mathrm{C}$ bilayer $\left(\mathrm{S}_{\mathrm{M}} \mathrm{C}_{2}\right)$, smectic A monolayer $\left(\mathrm{S}_{\mathrm{M}} \mathrm{A}_{1}\right)$, and smectic $\mathrm{C}$ monolayer $\left(\mathrm{S}_{\mathrm{M}} \mathrm{C}_{1}\right)$. Figure 10 sketches the different structures for each of these phases. The first structure arises in strongly segregated mesophases, where the high interfacial energy associated with rod-coil interfaces (given by a high $\chi$ ) has to be minimized by reducing the coil-rod interfacial area. This leads to a high energy of stretching of coil brush, partially released by the intercalated homopolymer rods, which essentially decreases the brush density. The last structure, the $\mathrm{S}_{\mathrm{M}} \mathrm{C}_{1}$, can only arise in a very weakly segregated regime. In this case, the interfacial energy is not minimized due to a tilt angle and to the presence of intercalated homopolymer rods, but for the same reasons, the stretching energy of the coil brush is greatly lowered by having low density of anchor sites. The other two structures correspond to weakly segregated regime, where, however, both interfacial area and coil stretching energy play a role. The specific model which suits best the cluster structure can therefore be assessed by calculating the thickness of the PS polymer brush in both weakly and strongly segregated regime as well as the thickness expected for the PPV
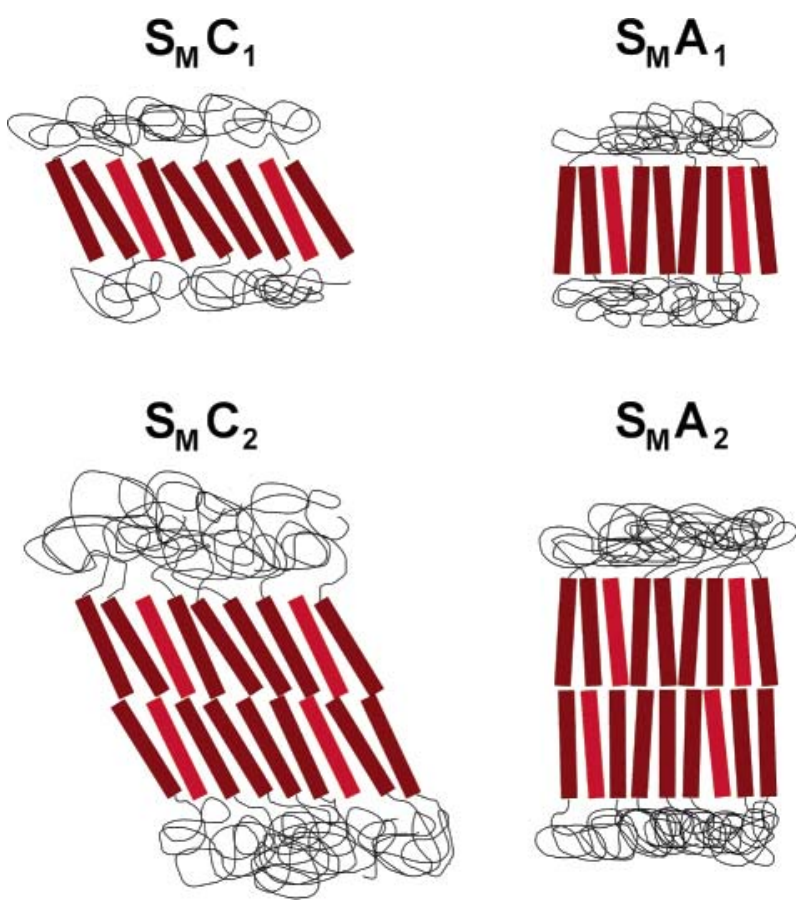

Figure 10. Possible molecular structures for the clusters observed in PPV/PS- $b$-PPV blends. The PPV block of PS- $b$-PPV block copolymer is represented as dark red rods, whereas the PPV homopolymer is in clear red. The ratio of PPV homopolymer/PPV block in the scheme is arbitrary. $\mathrm{S}_{\mathrm{M}} \mathrm{A}_{1}$ smectic A monolayer, $\mathrm{S}_{\mathrm{M}} \mathrm{A}_{2}$ smectic A bilayer, $\mathrm{S}_{\mathrm{M}} \mathrm{C}_{1}$ smectic $\mathrm{C}$ monolayer, $\mathrm{S}_{\mathrm{M}} \mathrm{C}_{2}$ smectic $\mathrm{C}$ bilayer.

domain. Given the delocalized $\pi$ bonds and considering the limited molecular weight, the persistence length of the PPV molecule is assumed to be larger than its contour length. In order to evaluate the phenylenevinylene monomer length, a conformational energy minimization using MOPAC algorithm was performed on a PPV trimer. The characteristic unit length $(l u)$, expressing the size of a PPV monomer, is then assumed to be the length obtained in the simulation for the trimer central unit. The rod length is calculated as the unit length $\left(l u_{\mathrm{PPV}}=\right.$ $0.660 \mathrm{~nm})$ times the number of units $\left(n_{\mathrm{PPV}}=9\right)$, determined by NMR, plus the end group size $\left(l_{\text {end }}=0.425 \mathrm{~nm}\right)$. Thus, the PPV contour length is defined as

$$
l_{\mathrm{PPV}}=n_{\mathrm{PPV}} l u_{\mathrm{PPV}}+l_{\mathrm{end}}
$$

By using eq 1, the PPV domain size is calculated to be 6.37 $\mathrm{nm}$ for the $\mathrm{S}_{\mathrm{M}} \mathrm{A}_{1}$ and $12.7 \mathrm{~nm}$ for the $\mathrm{S}_{\mathrm{M}} \mathrm{A}_{2}$ phases. For the rod diameter the experimental WAXS rod-to-rod distance is taken, $D_{\text {rod }}=0.96 \mathrm{~nm} \pm 1.4 \%$ (uncertainty margins are based on a normal distribution with $95 \%$ confidence).

The width of the PS domain $\left(D_{\mathrm{PS}}\right)$ in the strong segregation regime can be calculated on the basis of the law expressing the dependence of lamellae spacing and molecular weight for a poly(styrene- $b$-2-vinylpyridine) copolymer: ${ }^{41,42}$

$$
D_{\mathrm{PS}}=\frac{0.34\left(2 M_{\mathrm{n}, \mathrm{PS}}\right)^{0.64}}{2}
$$

This procedure was successfully applied in other PS-based rodcoil systems to estimate the thickness of the PS coil polymer domain. ${ }^{36}$

To estimate the polystyrene size domain in the limit of weakly segregated regime, one can take the exact half of the lattice parameter of a lamellar phase, formed by a symmetric block copolymer, in which one block is an identical PS block and the 
Table 1. Experimental and Theoretical Width of PS Domains, in the Weakly Segregated Regime (WSR) and Strong Segregated Regime (SSR); Experimental Width of PPV Domains Compared with Values Expected in a Smectic A Bilayer $\left(\mathbf{S}_{\mathbf{M}} \mathbf{A}_{2}\right)$ and Smectic A Monolayer $\left(S_{M_{1}} A_{1}\right)$ Configuration

\begin{tabular}{llll}
\hline & PS & \multicolumn{1}{c}{ PPV } \\
\hline SSR & $13.2 \mathrm{~nm}$ & single layer $\left(\mathrm{S}_{\mathrm{M}} \mathrm{A}_{1}\right)$ & $6.4 \mathrm{~nm}$ \\
WSR & $9.6 \mathrm{~nm}$ & double layer $\left(\mathrm{S}_{\mathrm{M}} \mathrm{A}_{2}\right)$ & $12.7 \mathrm{~nm}$ \\
exptl size: $e_{\mathrm{PS}}$ & $9.0 \mathrm{~nm}$ & measured size: $e_{\mathrm{PPV}}$ & $7.45 \pm 0.1 \mathrm{~nm}$
\end{tabular}

other block is made of a polymer having the same statistical segment length as PS. Following Leibler ${ }^{1}$ and Bates and Fredrickson, ${ }^{2}$ the period of the lamellar phase in such a case is $D_{\mathrm{PS}}=3.23 R_{\mathrm{g}}$, where $R_{\mathrm{g}}$ is the gyration radius of the whole block copolymer. Since the block copolymer is symmetric and PS makes up only half of its molecular weight, we can conclude that the depth of the PS domain in the rod-coil PPV-PS lamellae in a weakly segregated regime is

$$
D_{\mathrm{PS}}=\frac{3.23}{2} R_{\mathrm{g} 2}
$$

where $R_{\mathrm{g} 2}$ is the gyration radius of a block copolymer with molecular weight equal to the double of the one of PS. Since PS gyration radius is given by ${ }^{43}$

$$
\frac{R_{\mathrm{g}}^{2}}{M_{\mathrm{w}}}=0.0724
$$

a size of $9.6 \mathrm{~nm}$ is obtained for the PS domain in the clusters in the hypothesis of weakly segregated regime.

Table 1 summarizes the expected thickness of PS domains in the lamellae for weakly and strongly segregated regimes and also reports the thickness of the PPV domains in the case of $\mathrm{S}_{\mathrm{M}} \mathrm{A}_{1}$ and $\mathrm{S}_{\mathrm{M}} \mathrm{A}_{2}$ (Figure 10).

Comparison of the experimentally measured thickness of the PS domains $(9 \mathrm{~nm})$ agrees well with the PS width calculated in the weakly segregated regime $(9.6 \mathrm{~nm})$.

The measured value of the PPV domains in the clusters (7.4 $\mathrm{nm})$ is comprised between the theoretical value of monolayer $\mathrm{S}_{\mathrm{M}} \mathrm{A}_{1}(6.4 \mathrm{~nm})$ and that of bilayer $\mathrm{S}_{\mathrm{M}} \mathrm{A}_{2}(12.7 \mathrm{~nm})$. It may then be concluded that the rods are organized in a $\mathrm{S}_{\mathrm{M}} \mathrm{C}_{2}$ structure, which is the only one out of those sketched in Figure 10 giving values of PPV domains between $\mathrm{S}_{\mathrm{M}} \mathrm{A}_{1}$ and $\mathrm{S}_{\mathrm{M}} \mathrm{A}_{2}$ and which is also in accordance with a weakly segregated regime. Furthermore, tilted $\mathrm{S}_{\mathrm{M}} \mathrm{C}_{2}$ organization of the rods together with the presence of homopolymer rods in the PPV domains offers an additional route to decrease the density of anchor sites for the PS brush and thus the stretching energy of the chains.

The fact that the two blocks are weakly segregated is not surprising, since PS and PPV have rather similar chemical structures, and a thus very low $\chi$ is expected. The weakly segregated hypothesis is also supported by the low orderdisorder transition temperatures reported before.

As shown in Figure 11, the tilt angle of the $\mathrm{S}_{\mathrm{M}} \mathrm{C}_{2}$ can be easily determined on the basis of the measured PPV domain thickness, $e_{\mathrm{PPV}}$, and the calculated PPV contour length, $l_{\mathrm{PPV}}$ :

$$
\text { angle }=\arccos \left(\frac{e_{\mathrm{PPV}}}{2 l_{\mathrm{PPV}}}\right)=53.9^{\circ} \pm 1.5^{\circ}
$$

In order to minimize the distance of $\pi$-stacking interactions, only a limited number of positions will be allowed, that is, those positions at which two PPV units are facing each other. As a consequence, also the tilt angle will have a discrete set of

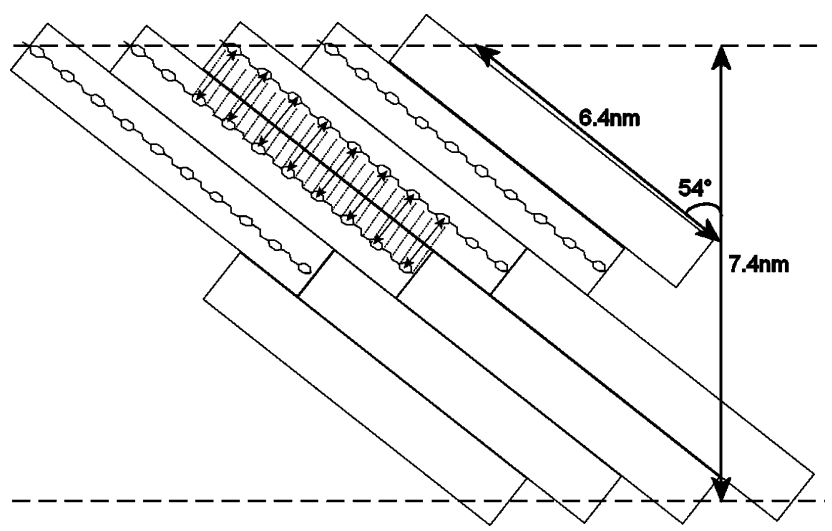

Figure 11. Schematic representation of the organization of the PPV rods within PPV cluster domains as resulting from WAXS data, molecular size calculation, and PPV width measured by TEM. The tilt angle of $54^{\circ}$ corresponds to a shift of the rods of two diethylhexyloxy$p$-phenylenevinylene units along the rod axis.

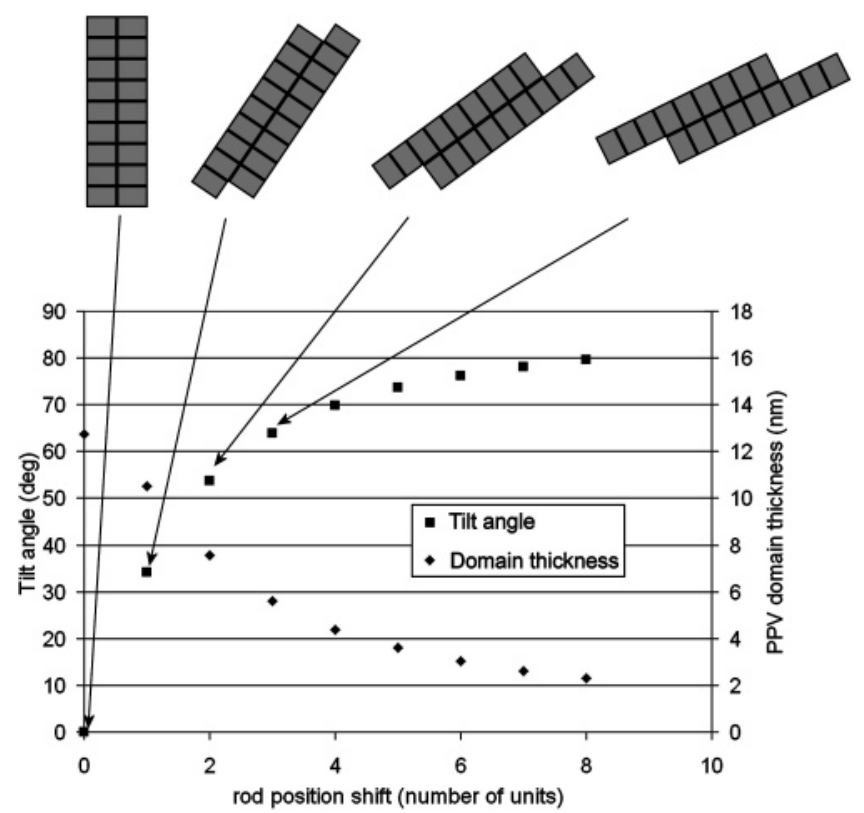

Figure 12. Quantization of the tilt angle and PPV domain width resulting from the $\pi-\pi$ optimum interactions. Upon minimization of the interdistance between diethylhexyloxy- $p$-phenylenevinylene units, only discrete tilt angles and PPV widths are allowed.

allowed values, which can be more formally expressed as

$$
\begin{gathered}
\text { tilt angle }=\arctan \left(\frac{n l u_{\mathrm{PPV}}}{D_{\mathrm{rod}}}\right) \quad n \in N \\
e_{\mathrm{PPV}}=2 l_{\mathrm{PPV}} \frac{D_{\mathrm{rod}}}{\sqrt{\left(n l u_{\mathrm{PPV}}\right)^{2}+D_{\mathrm{rod}}^{2}}}
\end{gathered}
$$

A quick calculation based on experimental $D_{\text {rod }}$ and monomer length unit obtained by simulations shows that, by taking $n$ equal to two PPV units, one recovers by eq 1 tilt angle ${ }_{(n=2)}=53.7^{\circ}$, which is exactly the experimentally determined angle in the $\mathrm{S}_{\mathrm{M}} \mathrm{C}_{2}$ clusters: $53.9 \pm 1.5^{\circ}$. In Figure 11, the quantization of the allowed angle is outlined by the overlap of the $\pi$-stacking, while Figure 12 illustrates the corresponding discrete widths of the PPV domains according to eq 6.

On the basis of the above considerations, the energetic driving force leading to the formation of the observed clusters can be identified as being made of three main contributions. 
The first two contributions are the energy associated with the stretching of PS polymer chains anchored at the PPV domains interface and the interfacial tension associated with the PPV/PS interfaces in the clusters. As discussed already by Halperin, ${ }^{44}$ rod may tilt, creating extra interfacial energy with subsequent increase in the interfacial energy contribution. At the same time the grafting density of PS chains is decreased by tilting, and thus, the stretching energy of PS brush is partially relaxed. The equilibrium conditions are those which minimize the total free energy based on these two contributions. In general, the lower the $\chi$, the larger will be the tilt angle, since the system can afford a greater increase of interfaces to relax the PS chain brush.

The presence of free rods in our case leads to a modification of the model first described by Halperin. First, the attractive stacking of free homopolymer rods to PPV block rods indicates that there is an enthalpic energy gain by close-packing the PPV rods. This gain will be proportional, in a first approximation, to the pair of PPV monomers in contact. Because the number of contacts is a function of the angle, as shown by eq 6 , the tilt will also have an influence on the energy gained by $\pi$-stacking. Very large tilt angles, leading to a low $\pi$-interaction exchange area, will reduce the energetic gain contribution associated with $\pi$-interactions. The energetic gain obtained by $\pi$-stacking will also have to be accounted as the third main contribution to the total free energy, together with chain stretching energy and interfacial energy. Finally, introducing free rods in the PPV domains also has an effect on the PS brush density, and by diluting the surface density of anchor sites, it constitutes an additional way to decrease the entropic penalty associated with stretched PS chains. A formal minimization of total free energy of the clusters leading to equilibrium tilted $\mathrm{S}_{\mathrm{M}} \mathrm{C}_{2}$ is presented in the Appendix.

Similarly to the asymmetric PS17K-PPV, the symmetric PS3K-PPV exhibited an isotropic phase where, however, disordered aggregated PPV domains could occasionally be observed, as shown in Figure 13a, driven by the larger PPV content. When the PS3K-PPV block copolymer was blended to PPV homopolymer of the same molecular weight of the rod block, nicely ordered lamellar clusters appeared, with long-range order maintained over several micrometers. Figure 13 shows the shape of lamellar clusters for a blends of PS3K-PPV and PPV at various concentrations of the PPV homopolymer rod. For low fraction of PPV homopolymer (5\%, Figure 13b) the lamellar clusters are still dispersed in a homogeneous PS3KPPV matrix and are thus unsuitable for optoelectronics applications requiring percolating interfaces and long-range order. However, upon blending of additional PPV homopolymer, in concentration between $10 \%$ and $25 \%$ (Figure 13c,d) lamellar clusters gain long-range order and percolate throughout the entire samples, thus fulfilling the requirement for optimal exciton dissociation at the interfaces. Further increase in PPV volume fraction leads to progressive macrophase separation of PPV and PS3K-PPV, as the contribution of the interfacial tension of free rods homopolymer in the clusters becomes too high to stabilize the clusters and the system better minimizes the total free energy by macroscopic phase separation.

The long-range order in these blends for optimal PPV homopolymer volume fraction can be understood considering the high equivalent volume fraction of PPV in the blend. Owing to the large volume fraction of the clusters in PS3K-PPV:PPV blends the structure period could be determined by SAXS. This was found to be $11 \mathrm{~nm}$ for blends of $10 \mathrm{wt} \%$ homopolymer$90 \%$ block copolymer without significant variation in the
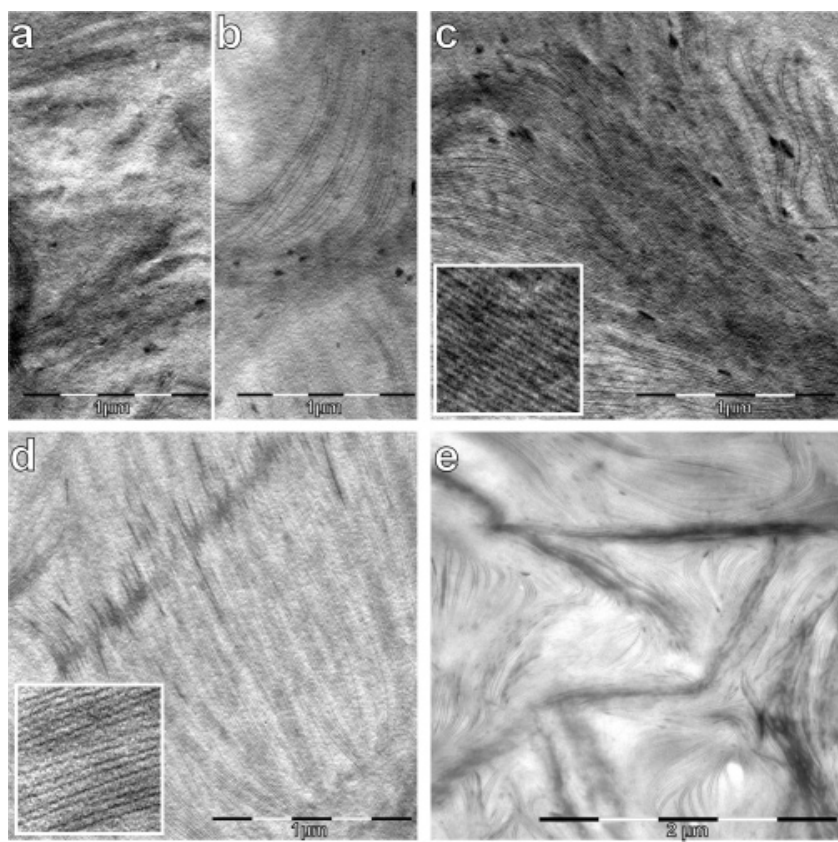

Figure 13. (a) TEM micrograph of PS3K-PPV showing disordered aggregates of PPV sparsely dispersed in an isotropic PPV-PS continuous phase. (b) When 5\% PPV homopolymer is added to $95 \%$ PS3K-PPV localized lamellar clusters start to appear, although these remain disperse in a continuous isotropic phase. When (c) $10 \mathrm{wt} \%$ (inset has $200 \mathrm{~nm}$ edge) and (d) $25 \mathrm{wt} \%$ (inset has $200 \mathrm{~nm}$ edge) PPV homopolymer are added to the blend, however, lamellar clusters with long-range order percolating throughout the entire sample are formed. Upon further increase on volume fraction of the PPV homopolymer, macrophase separation starts to occur, as shown in (e), where the morphology of a $40 \%$ PPV $60 \%$ PS3K-PPV is shown.

temperature range from 120 to $180{ }^{\circ} \mathrm{C}$. This indicates that (i) the tilt angle is temperature independent of this system and that (ii) the order-disorder temperature of the clusters in PS3KPPV:PPV is well above the temperature region which can be explored without thermal degradation of either block. Similarly to the clusters observed in the PS17K-PPV:PPV blend, also in this case the clusters were found to be organized in a $\mathrm{S}_{\mathrm{M}} \mathrm{C}_{2}$ topology with a tilt angle of $51.8 \pm 2.3^{\circ}$, which is very close to the one observed in PS17K-PPV:PPV clusters. These results are at first sight counterintuitive as one would expect a lower tilt angle based on the shorter PS brush, as compared to the PS17K-PPV case. However, in the PS3K-PPV block copolymer, the PPV rods being shorter, the negative $\pi$-stacking contribution to the total free energy, which tends to stabilize lower tilt angle is reduced compared to PS17K-PPV, which can explain values of the tilt angle comparable for the PS17KPPV:PPV and PS3K-PPV:PPV blends.

\section{Conclusions}

The self-assembly behavior in blends of $\pi$-conjugated poly(diethylhexyloxy-p-phenylenevinylene) rod homopolymers (PPV) and poly(diethylhexyloxy- $p$-phenylenevinylene)-polystyrene (PPV- $b$-PS) rod-coil block copolymers has been described. Although the rod-coil alone forms a homogeneous isotropic phase, ordered clusters of PPV domains alternated by PS domains appeared upon blending of PPV and PPV- $b$-PS. The exact structure of the clusters was assessed by tomographic electron transmission microscopy, which allowed determining the lamellar nature of the clusters by tilting microtomed sections of the blend at different angles. Wide-angle X-ray scattering revealed the presence of rod-rod order in the PPV domains with inter-rod distance of $1 \mathrm{~nm}$. 
By resolving the width of PS and PPV domains in the clusters and by calculating the expected width of the PS domain in both weakly and strongly segregated regime, it was possible to determine the exact molecular organization of the lamellar clusters, which was shown to be consistent with weakly segregated smectic $C$ bilayers $\left(\mathrm{S}_{\mathrm{M}} \mathrm{C}_{2}\right)$, in which the PPV domains are formed by intercalated homopolymer and block rods, tilted by $54^{\circ}$ with respect to the normal to the lamellar plane. Calculation on the molecular packing of the PPV rods indicated that the tilting angle of $54^{\circ}$ corresponds to a systematic shift of rod alignment of two diethylhexyloxy- $p$-phenylenevinylene units. The morphology observed for the $\mathrm{S}_{\mathrm{M}} \mathrm{C}_{2}$ lamellar clusters suggested that these structures originate as a result of the minimization of the free energy based on three major energetic contributions: the interfacial energy between PPV rods and PS chains, the stretching energy of the PS chains, and the $\pi$-stacking enthalpic energy gain arising from PPV rods interactions. In practice, the presence of PPV homopolymer rods in the PPV clusters domains decreases the stretching energy of PS polymer brush and increases the $\pi$-stacking energy gain at the expenses of PPV-PS interfacial energy. Equivalently, the PPV intercalation can be viewed as increasing the equivalent volume fraction of the rod in the block copolymer, thus driving the equilibrium structure of the rod-coil block copolymers from an isotropic fluid to a lamellar morphology. These concepts were used to ultimately design long-range order lamellar clusters percolating throughout the entire samples when PPV homopolymer rods were blended with symmetric PS-PPV rod-coil block copolymers. Therefore, the blending procedure of rod homopolymer/rod-coil block copolymer described on this paper is bound to have an impact for the fine control and design of interfaces in organic optoelectronic devices based on selfassembly of rod-coil block copolymers, and this study has also relevance for those synthetic routes for which potential structural effects may arise owing to residual presence of conjugated homopolymer in the block copolymer synthetic batch.

Acknowledgment. This work was supported by the Swiss Science National Fundation and BASF Aktiengesellschaft. We thank David Yip from Stanford University for his help and efficient support of the EM3D software.

\section{Appendix}

The energy contributions related to the PS/PPV interfacial tension and the stretching of PS chains have been already discussed in the literature and are expressed respectively as ${ }^{44}$

$$
\begin{aligned}
\frac{F_{\text {interface }}}{k T} & =\frac{\gamma d^{2}}{k T}(1+|\tan \theta|) \\
\frac{F_{\text {corona }}}{k T} & =N\left(\frac{a}{d}\right)^{5 / 3} \cos ^{5 / 6} \theta
\end{aligned}
$$

where $a$ is the styrene typical size, $d$ is the diameter of a PPV rod, $N$ is the number of PS units, and $\gamma$ the PS/PPV interfacial tension. The calculation of the energy gain arising from $\pi$-stacking interaction is a very complex task since this interaction is not pairwise but is delocalized to many stacked layers. ${ }^{45}$ In order to illustrate the influence of this contribution, as a first, very rough approximation one can estimate this energy as being directly proportional to the exchange surface area in the PPV domains. In this case, it can be written

$$
\frac{F_{\pi-\pi}}{k T} \approx A L\left[2 l_{\mathrm{PPV}}-2 d \tan (90-\theta)^{-1}\right]
$$

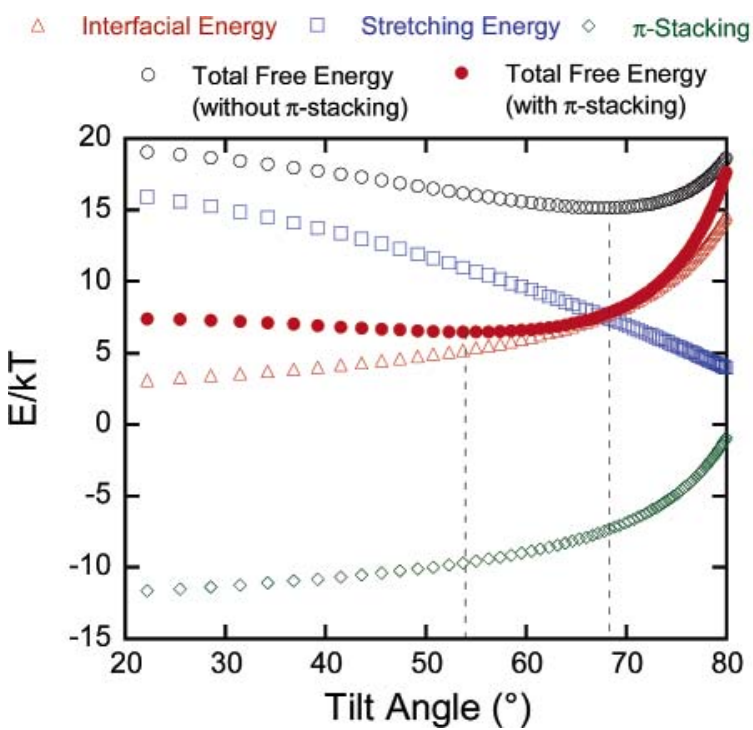

Figure 14. (i) PS/PPV interfacial energy, (ii) stretching energy of PS chains, and (iii) $\pi$-stacking energetic gain as a function of the tilt angle. The total free energy is illustrated based on (i) plus (ii) contributions and (i) plus (ii) plus (iii) contributions. Including the $\pi$-stacking energetic gain in the classical energetic calculation (performed by Halperin), shifts the minimum of the total free energy toward lower tilting angles of the rods.

where $A$ is a negative constant dependent on the strength of $\pi$-stacking and $L$ is the characteristic length of the cluster lamellae perpendicular to the section of Figure 11.

The equilibrium tilting angle can be obtained by minimizing as a function of $\theta$ the sum of (A1), (A2), and (A3) contributions, once all prefactors are known. In plotting Figure 14, we have fixed for $\gamma$ and $A L$ values yielding a minimum of total free energy at $54^{\circ}$.

\section{References and Notes}

(1) Leibler, L. Macromolecules 1980, 13, 1602-1617.

(2) Bates, F. S.; Fredrickson, G. H. Annu. Rev. Phys. Chem. 1990, 41, 525-557.

(3) Bates, F. S.; Fredrickson, G. H. Phys. Today 1999, 52, 32-38.

(4) Friedel, P.; John, A.; Pospiech, D.; Jehnichen, D.; Netz, R. R. Macromol. Theory Simul. 2002, 11, 785-793.

(5) Winey, K. I.; Thomas, E. L.; Fetters, L. J. Macromolecules 1992, 25, 2645-2650.

(6) Hadziioannou, G.; Skoulios, A. Macromolecules 1982, 15, 267-271.

(7) de Boer, B.; Stalmach, U.; van Hutten, P. F.; Melzer, C.; Krasnikov, V. V.; Hadziioannou, G. Polymer 2001, 42, 9097-9109.

(8) Segalman, R. A.; Brochon, C.; Hadziioannou, G.; Sun, S.-S.; Sariciftci, N. S., Eds.; Taylor \& Francis: London, 2005; p 403.

(9) van der Veen, M. H.; de Boer, B.; Stalmach, U.; van de wetering, K. I.; Hadziioannou, G. Macromolecules 2004, 37, 3673-3684.

(10) Iovu, M. C.; Jeffries-El, M.; Sheina, E. E.; Cooper, J. R.; McCullough, R. D. Polymer 2005, 46, 8582-8586.

(11) Liu, J. S.; Sheina, E.; Kowalewski, T.; McCullough, R. D. Angew. Chem., Int. Ed. 2001, 41, 329.

(12) Marsitzky, D.; Klapper, M.; Mullen, K. Macromolecules 1999, 32, 8685-8688.

(13) Lu, S.; Fan, Q. L.; Chua, S. J.; Huang, W. Macromolecules 2003, 36, 304-310.

(14) Van De Wetering, K.; Brochon, C.; Ngov, C.; Hadziioannou, G. Macromolecules 2006, 39, 4289-4297.

(15) Tsolakis, P. K.; Kallitsis, J. K.; Godt, A. Macromolecules 2002, 35, 5758-5762.

(16) Chochos, C. L.; Kallitsis, J. K.; Gregoriou, V. G. J. Phys. Chem. B 2005, 109, 8755-8760.

(17) Leclere, P.; Parente, V.; Bredas, J. L.; Francois, B.; Lazzaroni, R. Chem. Mater. 1998, 10, 4010-4014.

(18) Marsitzky, D.; Brand, T.; Geerts, Y.; Klapper, M.; Mullen, K. Macromol. Rapid Commun. 1998, 19, 385-389.

(19) Francke, V.; Rader, H. J.; Geerts, Y.; Mullen, K. Macromol. Rapid Commun. 1998, 19, 275-281. 
(20) Hempenius, M. A.; Langeveld-Voss, B. M. W.; van Haare, J.; Janssen, R. A. J.; Sheiko, S. S.; Spatz, J. P.; Moller, M.; Meijer, E. W. J. Am. Chem. Soc. 1998, 120, 2798-2804.

(21) Kukula, H.; Ziener, U.; Schops, M.; Godt, A. Macromolecules 1998, $31,5160-5163$.

(22) Wang, H. B.; Wang, H. H.; Urban, V. S.; Littrell, K. C.; Thiyagarajan, P.; Yu, L. P. J. Am. Chem. Soc. 2000, 122, 6855-6861.

(23) Li, W. J.; Wang, H. B.; Yu, L. P.; Morkved, T. L.; Jaeger, H. M. Macromolecules 1999, 32, 3034-3044.

(24) Tew, G. N.; Pralle, M. U.; Stupp, S. I. J. Am. Chem. Soc. 1999, 121, 9852-9866.

(25) Olsen, B. D.; Segalman, R. A. Macromolecules 2005, 38, 1012710137.

(26) Szwarc, M. J. Polym. Sci., Part A: Polym. Chem. 1998, 36, IX-XV.

(27) Semenov, A. N. Mol. Cryst. Liq. Cryst. 1991, 209, 191-199.

(28) De Gennes, P. G. The Physics of Liquid Crystal; Clarendon Press: Oxford, 1975.

(29) Lee, M.; Oh, N. K. J. Mater. Chem. 1996, 6, 1079-1086.

(30) Lee, M.; Cho, B. K.; Kang, Y. S.; Zin, W. C. Macromolecules 1999, 32, 7688-7691.

(31) Lee, M.; Cho, B. K.; Kim, H.; Yoon, J. Y.; Zin, W. C. J. Am. Chem. Soc. 1998, 120, 9168-9179.

(32) Reenders, M.; ten Brinke, G. Macromolecules 2002, 35, 3266-3280.
(33) Matsen, M. W.; Barrett, C. J. Chem. Phys. 1998, 109, 4108-4118.

(34) Williams, D. R. M.; Fredrickson, G. H. Macromolecules 1992, 25, $3561-3568$.

(35) Chen, J. T.; Thomas, E. L.; Ober, C. K.; Hwang, S. S. Macromolecules 1995, 28, 1688-1697.

(36) Chen, J. T.; Thomas, E. L.; Ober, C. K.; Mao, G. P. Science 1996, 273, 343-346.

(37) Heeger, A. J. Rev. Mod. Phys. 2001, 73, 681-700.

(38) Stalmach, U.; de Boer, B.; Post, A. D.; van Hutten, P. F.; Hadziioannou, G. Angew. Chem., Int. Ed. 2001, 40, 428-430.

(39) Ress, D.; Harlow, M. L.; Schwarz, M.; Marshall, R. M.; McMahan, U. J. J. Electron Microsc. 1999, 48, 277-287.

(40) Balaban, T. S.; Eichhofer, A.; Lehn, J. M. Eur. J. Org. Chem. 2000, 4047-4057.

(41) Matsushita, Y.; Mori, K.; Saguchi, R.; Nakao, Y.; Noda, I.; Nagasawa, M. Macromolecules 1990, 23, 4313-4316.

(42) Hadziioannou, G.; Skoulios, A. Macromolecules 1982, 15, 258-262.

(43) Brulet, A.; Boue, F.; Cotton, J. P. J. Phys. II 1996, 6, 885-891.

(44) Halperin, A. Macromolecules 1990, 23, 2724-2731.

(45) Ye, X. Y.; Li, Z. H.; Wang, W. N.; Fan, K. N.; Xu, W.; Hua, Z. Y. Chem. Phys. Lett. 2004, 397, 56-61.

MA0628300 